\title{
Cefiderocol, a New Siderophore Cephalosporin for the Treatment of Complicated Urinary Tract Infections Caused by Multidrug-Resistant Pathogens: Preclinical and Clinical Pharmacokinetics, Pharmacodynamics, Efficacy and Safety
}

\author{
Young Ran Lee ${ }^{1}\left[\right.$ Suyeon Yeo $^{2}$ (D)
}

Published online: 22 July 2020

(c) Springer Nature Switzerland AG 2020

\begin{abstract}
Cefiderocol (Fetroja $\left.{ }^{\circledR}\right)$ is a siderophore cephalosporin and has demonstrated potent activity against extended-spectrum betalactamases producing Enterobacteriaceae, carbapenem-resistant Enterobacteriaceae, and nonfermenting Gram-negative bacilli, including Pseudomonas aeruginosa, Stenotrophomonas maltophilia, and Acinetobacter baumannii, Burkholderia cepacia, and Klebsiella pneumoniae. However, cefiderocol has limited activity against Gram-positive bacteria and anaerobes like Bacterodies fragilis. In the APEKS-cUTI study, 183 (73\%) of 252 patients in the cefiderocol group versus $65(55 \%)$ of 119 patients in the imipenem-cilastatin group achieved the composite outcome of clinical and microbiological eradication of Gram-negative bacteria (treatment difference of $18.58 \%$; 95\% CI 8.23-28.92, $\mathrm{p}=0.0004$ ) in complicated urinary tract infections (cUTIs). Cefiderocol was non-inferior to imipenem-cilastatin in cUTIs caused by Gram-negative bacteria such as E. coli, K. pneumoniae, P. aeruginosa, Proteus mirabilis, Enterobacter cloacae, Morganella morganii, and Citrobacter freundii. Cefiderocol required dose adjustment in patients with renal impairment and percentage of time that free drug concentrations above the minimum inhibitory concentration (\% $\mathrm{fT}>\mathrm{MIC}$ ) best correlated with clinical outcomes. The most common adverse events with cefiderocol were gastrointestinal symptoms such as diarrhea, constipation, nausea, vomiting, or upper abdominal pain. Two phase III clinical trials, the CREDIBLE-CR study and the APEKS-NP study, investigated the efficacy and safety of cefiderocol for the treatment of pneumonia or cUTI, and both studies showed higher all-cause mortality associated with cefiderocol. Therefore, the use of cefiderocol should be limited only to the treatment of cUTIs from Gramnegative bacteria, especially in patients who have limited or no alternative treatment options.
\end{abstract}

\section{Introduction}

According to the Centers for Disease Control and Prevention (CDC), infections caused by carbapenem-resistant Enterobacteriaceae (CRE) organisms are associated with high mortality rates in inpatient healthcare settings up to 50\% [1]. CRE can result in bloodstream infections, ventilator-associated pneumonia, intra-abdominal abscesses, and urinary

Young Ran Lee

young.lee@ttuhsc.edu

1 Department of Pharmacy Practice, Adult Medicine Division, Jerry H. Hodge School of Pharmacy, Texas Tech University Health Sciences Center, 1718 Pine Street, Abilene, TX 79601, USA

2 Jerry H. Hodge School of Pharmacy, Texas Tech University Health Sciences Center, Amarillo, TX, USA tract infections due to exposure to healthcare and antibiotics $[1,2]$. Hence, it is imperative to develop a new antibiotic to overcome antimicrobial resistance. Cefiderocol was developed in line with US Food and Drug Administration (FDA)guided streamlined development for antibiotics to treat lifethreatening infections caused by multidrug-resistant (MDR) Gram-negative bacteria (GNB) [3]. Cefiderocol, a new parenteral siderophore cephalosporin, has demonstrated potent activities against Gram-negative pathogens such as carbapenem-resistant Enterobacteriaceae, Pseudomonas aeruginosa, Acinetobacter baumannii, Burkholderia cepacia, and Stenotrophomonas maltophilia that are MDR and carbapenem-resistant isolates by producing carbapenemases and $\beta$-lactamases [4-7]. Cefiderocol is approved for the treatment of complicated urinary tract infection (cUTI) with limited or no alternative treatment options [8]. 


\section{Key Points}

Cefiderocol $\left(\right.$ Fetroja $\left.^{\circledR}\right)$ is a siderophore cephalosporin that results in an iron-depleted environment of Gramnegative bacteria via the "Trojan horse" strategy.

Cefiderocol has demonstrated time-dependent bactericidal activity against Gram-negative bacteria, especially those with multidrug-resistant organisms, including carbapenem-resistant Enterobacteriaceae (CRE).

Cefiderocol has received the US Food and Drug Administration approval for the treatment of complicated urinary tract infections (cUTIs) from Gram-negative bacteria, especially in patients who have limited or no alternative treatment options.

Due to an increase in all-cause mortality in patients treated with cefiderocol compared to those treated with best available therapy, cefiderocol is reserved for cUTIs in carbapenem-resistant Gram-negative bacterial infections.

\section{Methods of Literature Review}

A literature search from PubMed (1996-March 2020) and EMBASE (1947-March 2020) was conducted using terms including "cefiderocol," "S-649266," or "multi-drug resistant pathogens." Results were limited to primary literature published in English. Additional information was gathered from Shionogi Inc. website and clinicalTrials.gov. provided by the US National Library of Medicine.

\section{Mechanism of Action}

Cefiderocol is a siderophore cephalosporin with a catechol group (Fig. 1), which is important for antibacterial activities against multi-drug resistant GNB such as $P$. aeruginosa and $A$. baumannii $[5,9]$. The carboxylic acid of the C-7 side chain improves cefiderocol permeability into the outer membrane, and the chlorocatechol group of the C-3 side chain chelates iron. This chelating activity is responsible for antibacterial activities of cefiderocol, resulting in an irondepleted environment and the uptake of cefiderocol [10]. By inhibiting mainly penicillin-binding protein 3 (PBP3) of Enterobacteriaceae and nonfermenting GNB, the antibacterial action of cefiderocol results in morphological changes of filamentous cells [4]. Active transport of cefiderocol into GNB and its stability against clinically relevant carbapenemases have shown a dual antimicrobial activity of this siderophore cephalosporin [10].

\section{Spectrum of Activity}

Cefiderocol has demonstrated potent activity against a range of GNB such as Enterobacteriaceae, A. baumannii, P. aeruginosa, B. cepacia, and S. maltophilia, including MDR and carbapenem-resistant isolates that produce carbapenemases and $\beta$-lactamases $[6,7]$. In the SIDERO-CR study, cefiderocol demonstrated efficacy against isolates of Gram-negative bacilli that are not susceptible to ceftazidime-avibactam, ceftolozane-tazobactam, and colistin [11]. Compared to cefepime or meropenem, cefiderocol has shown weak activity against multiple clinical isolates of anaerobic bacteria such as Bacteroides spp., Prevotella spp., or Clostridioides difficile [4]. The antibacterial activity of cefiderocol was superior to that of comparators, including cephalosporins, carbapenems, fluoroquinolones, and monobactams against MDR Gram-negative pathogens, except for colistin and tigecycline, with equivalent efficacy against certain subgroup organisms [12]. However, another study found that cefiderocol showed lower minimum inhibitory concentrations (MICs) compared to ten antibiotics against carbapenem-resistant GNB, including strains resistant to colistin and aminoglycoside. It has demonstrated that isolates producing carbapenemases or $\beta$-lactamases cannot develop resistance to cefiderocol [13]. Seven studies investigated in vitro activities of cefiderocol against MDR Gram-negative pathogens. Dobias et al. demonstrated excellent cefiderocol activity against $K$. pneumoniae carbapenemase (KPC), OXA, inosine-5'-monophosphate (IMP), Verona integronmediated metallo- $\beta$-lactamase (VIM), New Delhi metallo$\beta$-lactamases (NDM)-producing Enterobacteriaceae and carbapenemase- [IMP, KPC, VIM, São Paulo metallo- $\beta$ lactamase (SPM), Germany imipenemase (GIM)] producing $P$. aeruginosa [12]. Kazmierczak et al. demonstrated

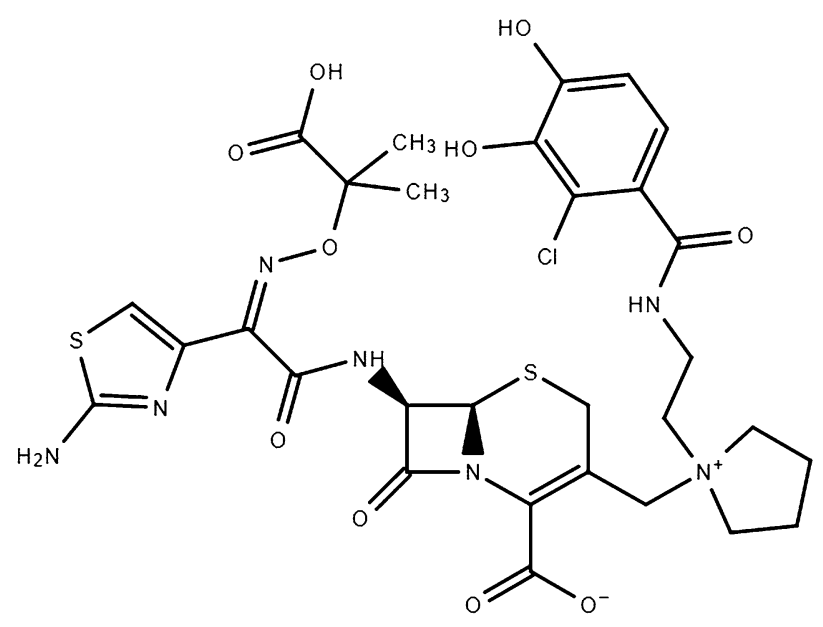

Fig. 1 The chemical structure of cefiderocol 
excellent cefiderocol activity against IMP, OXA, KPC, VIM, and NDM producing resistant Enterobacteriaceae [14]. Jacobs et al. demonstrated excellent cefiderocol activity against MDR A. baumannii, S. maltophilia, P. aeruginosa, and Enterobacteriaceae [15]. Cefiderocol demonstrated potent in vitro activity against 231 isolates of MDR GNB, and $98 \%$ of which were MICs of $4 \mathrm{mg} / \mathrm{L}$ shown to be superior to comparators [16]. Five isolates were not susceptible to cefiderocol (MIC $>4 \mathrm{mg} / \mathrm{L}$ ), including three ST2/OXA24/40-producing A. baumannii, one ST114/VIM-1-producing E. cloacae, one ST114/VIM-1, and OXA-48-producing E. cloacae [16]. All KPC-3-producing K. pneumoniae resistant to ceftazidme/avibactam were inhibited by cefiderocol (MIC $<4 \mathrm{mg} / \mathrm{L}$ ). P. aeruginosa and S. maltophilia isolates were inhibited by cefiderocol (both at MICs $<4 \mathrm{mg} / \mathrm{L}$ ), which were not susceptible to ceftolozane/tazoactam and levofloxacin, respectively [16]. Golden et al. reported that all 800 isolates of Gram-negative bacilli from intensive care units (ICUs), including of extended-spectrum $\beta$-lactamases (ESBL)-producing $(n=40)$, AmpC-producing $(n=6)$, and carbapenem-nonsusceptible $(n=21)$ Enterobacterales, carbapenem-nonsusceptible $(n=54)$ and MDR $(n=29)$ $P$. aeruginosa, $S$. maltophilia $(n=66)$, and A. baumannii $(n=11)$ were susceptible to cefiderocol with an MIC $\leq 4 \mu \mathrm{g} / \mathrm{mL}$ of which $99 \%(600 / 606)$ met FDA breakpoints of MIC $\leq 2 \mu \mathrm{g} / \mathrm{mL}$ [17]. Notably, cefiderocol demonstrated a fourfold potent antimicrobial activity to $P$. aeruginosa than compactors, including ceftazidime/avibactam, ceftolozane/ tazobactam, colistin, meropenem, and piperacillin/tazobactam, which is a pathogen responsible for pneumonia in critically ill patients in ICUs [17]. Cefiderocol had potent in vitro activity against $97.5 \%$ of 478 GNB isolates from cancer patients with MIC90 $\leq 4 \mathrm{mg} / \mathrm{L}$ [18]. It has demonstrated activity against isolates of ESBL-positive Enterobacteriaceae, CRE, and nonfermenting Gram-negative bacilli, including P. aeruginosa, S. maltophilia, and Acinetobacter [18]. Against non-CRE, ceftazidime-avibactam, meropenem, and tigecycline had a comparable activity to cefiderocol; however, cefiderocol alone was active against MDR $P$. aeruginosa isolates [18]. Cefiderocol and trimethoprimsulfamethoxazole had appreciable activity against $S$. maltophilia isolates [18]. Overall, cefiderocol demonstrated the lowest level of resistance to GNB [18].

While neither resistance pattern to cefiderocol nor the underlying mechanisms have been studied, Kawai et al. reported reduced susceptibility to cefiderocol in AmpC betalactamases with R2 loop deletion that increased hydrolysis of cefiderocol and ceftazidime-avibactam [19]. This finding explained the evolving survival strategy of MDR GNB to beta-lactam agents under selective pressure, and warrants further studies [19].

Table 1 summarizes data of the MIC50 and MIC90 of the antibiotics against bacterial isolates.

\section{Pharmacokinetics and Pharmacodynamics}

A phase I, randomized, double-blind, placebo-controlled study was conducted to evaluate the pharmacokinetics, safety, and tolerability of cefiderocol in healthy subjects [20]. A single ascending-dose study included doses of 100 , $250,500,1,000$, or $2000 \mathrm{mg}$ cefiderocol, and a multiple ascending-dose study evaluated doses of 1000 and $2000 \mathrm{mg}$ cefiderocol every $8 \mathrm{~h}$ over 60 -min intravenous infusions [20]. Dose-dependent increases appeared in the maximum plasma concentration $\left(C_{\max }\right)$ and area under the plasma concentration-time curve (AUC) following single and multiple intravenous infusions of 100-2000 mg [20]. This study demonstrated that cefiderocol was safe and well tolerated in patients administered $2000 \mathrm{mg}$ every $8 \mathrm{~h}$ [20]. No clinically significant adverse reactions were reported except one patient receiving $1000 \mathrm{mg}$ every $8 \mathrm{~h}$ was withdrawn due to fever [20]. Cefiderocol was excreted in the urine mostly unchanged (60-70\%), and no accumulation of cefiderocol and cefiderocol metabolites were observed after a 1-h intravenous infusion of $1000 \mathrm{mg}$ cefiderocol in healthy subjects $[20,21]$. Pharmacokinetic parameters including $C_{\max }$, AUC, total clearance $(\mathrm{CL})$, and terminal half-life $\left(t_{1 / 2}\right)$ of the multiple dosing every $8 \mathrm{~h}$ were similar to those of the single-dose study, indicating there was no change in pharmacokinetics [20].

Since most cefiderocol is renally excreted, another study evaluated pharmacokinetics and safety of cefiderocol in subjects with renal impairment [22]. A single intravenous infusion of $1000 \mathrm{mg}$ over $1 \mathrm{~h}$ was administered to subjects with mild, moderate, or severe renal impairment and endstage renal disease (ESRD) with or without hemodialysis [22]. Cefiderocol was given twice for subjects with hemodialysis-dependent ESRD [22]. In comparison to a normal renal function group, there was increased cefiderocol exposure in subjects with moderate, severe renal impairment and those with ESRD without hemodialysis, as evidenced by increased AUC and the mean plasma $t_{1 / 2}$ [22]. The study found that renal impairment impacted AUC, CL, and $t_{1 / 2}$ with little change in $C_{\max }$ [22]. Ratios of AUC in mild, moderate, severe, and ESRD groups to those with normal renal function were 1.0, 1.5, 2.5, and 4.1, respectively; however, ratios of $C_{\max }$ were similar between groups, as shown 0.9, $0.9,1.0$, and 1.1, respectively [22]. Due to the significant removal of cefiderocol with hemodialysis, dose adjustment is suggested in patients with renal impairment [22].

Pharmacokinetic/pharmacodynamic modeling and simulation of cefiderocol were conducted to determine dose adjustment based on renal function [23]. Using plasma, urine, and dialysate data in two phase I studies, population pharmacokinetic models were proposed using a nonlinear mixed-effect model [23]. The pharmacodynamic index, 


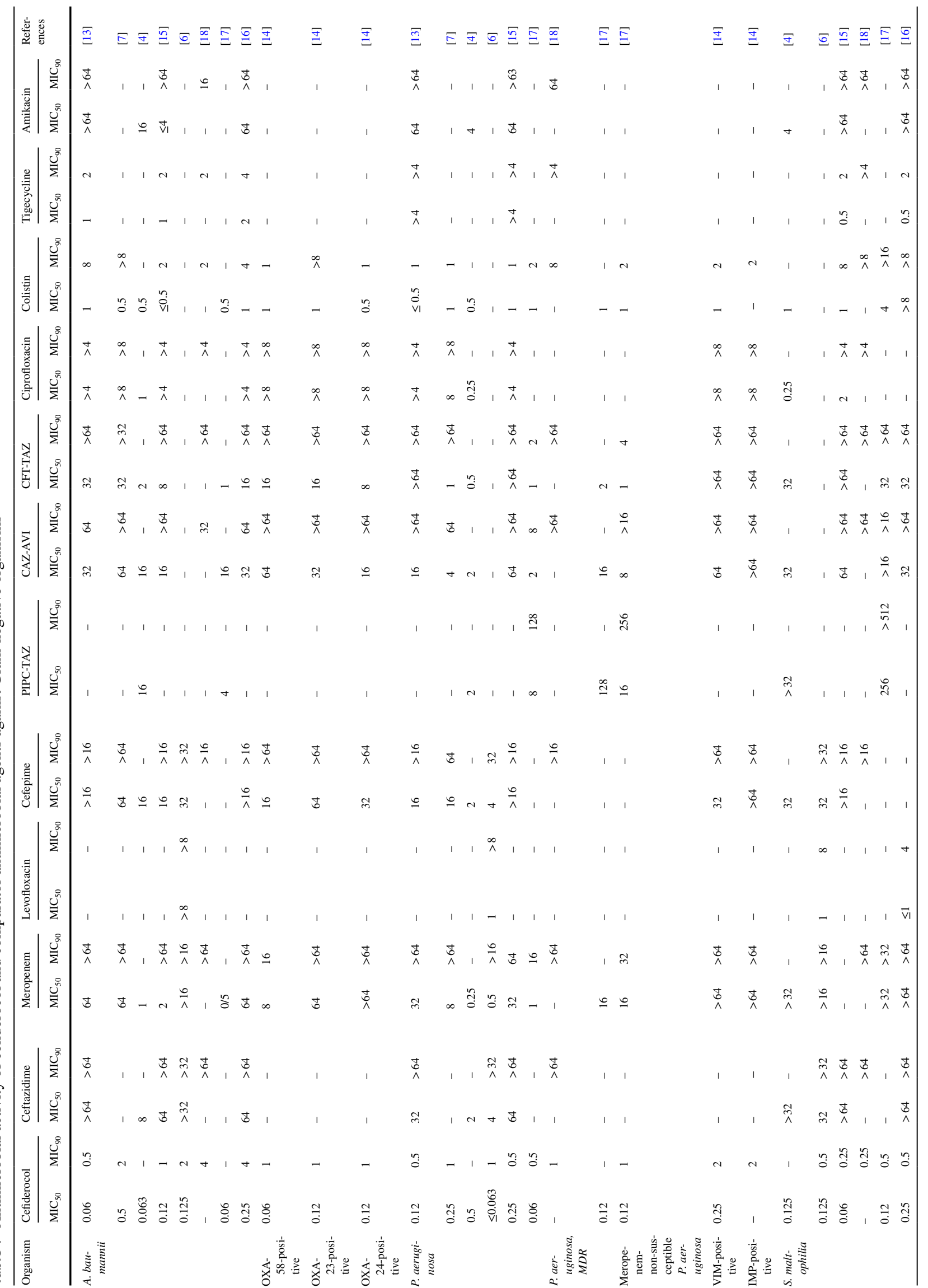




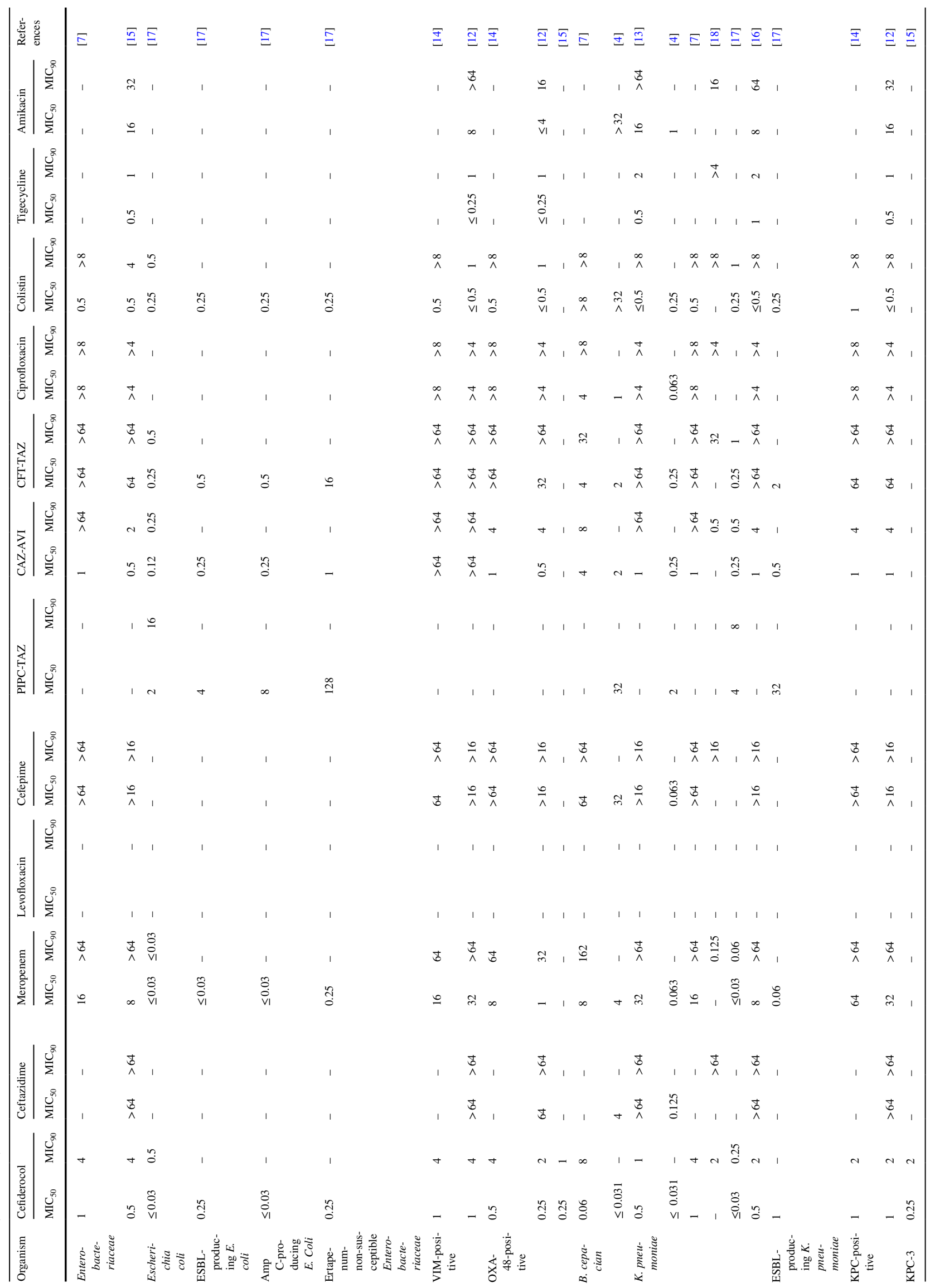


percentage of time that free drug concentrations above the minimum inhibitory concentration (\% fT > MIC), was best correlated with clinical outcomes [8]. Hence, Monte-Carlo simulations were used to calculate the probability of target attainment (PTA) for the MIC range of $0.25-16 \mu \mathrm{g} / \mathrm{mL}$ [23]. Adjusted dose regimens were determined based on $C_{\max }$ and daily AUC per renal function group using the MDRD-eGFR covariate model [23]. For subjects with normal renal function and mild renal impairment, a dose of 2,000 mg cefiderocol every $8 \mathrm{~h}$ with 3 -h infusion achieved $90 \%$ PTA where $75 \%$ fraction of time was against strains with MIC $<4 \mu \mathrm{g}$ $/ \mathrm{mL}$ [23]. More frequent dosing every $6 \mathrm{~h}$ is desirable for patients with augmented renal function based on CG-CL ${ }_{\mathrm{CR}}$ [23]. Moderate and severe renal impairment requires 1.5 and $1 \mathrm{~g}$, respectively, every $8 \mathrm{~h}$ with 3 -h infusion; $0.75 \mathrm{~g}$ every $12 \mathrm{~h}$ with 3 -h infusion in ESRD patients with or without hemodialysis [23].

A summary of pharmacokinetic data is available in Table 2 .

\section{Efficacy}

\subsection{In Vitro Activity}

Six in vitro studies have been conducted to demonstrate the antimicrobial activity of cefiderocol against isolates, including MDR and carbapenem-resistant GNB [7, 11, 13, 24]. In the SIDERO-WT-2014 study, MIC to inhibit the growth of 90\% isolates (MIC90) was determined for the 9,205 clinical isolates of GNB, such as Enterobacteriaceae, $P$. aeruginosa, A. baumannii, S. maltophilia, and B. cepacian from North America and Europe [24]. In this study, the in vitro activity of cefiderocol was superior to that of antibiotic comparators, including colistin, ceftazidime-avibactam, and ceftolozanetazobactam against isolates of meropenem-resistant and MDR GNB, which limits current treatment options for MDR infections [24]. Cefiderocol was effective against isolates of CRE, MDR P. aeruginosa, MDR A. baumannii, S. maltophilia, and B. cepacian, as evidenced by MICs $\leq 4 \mu \mathrm{g} / \mathrm{mL}$ $[11,24]$.

In another study comparing the efficacy of cefiderocol against MDR Gram-negative isolates with comparators, including ceftolozane-tazobactam (CT), meropenem (MEM), ceftazidime (CAZ), ceftazidime-avibactam (CZA), colistin (CST), aztreonam (ATM), amikacin (AMK), ciprofloxacin (CIP), cefepime (FEP), and tigecycline (TGC) [8]. Cefiderocol (MIC90 2-4 mg/L) demonstrated more potent efficacy compared to other comparators (MIC90 > 4 to $>64$ $\mathrm{mg} / \mathrm{L}$ ) against all isolates, except colistin and tigecycline, having comparable efficacy to cefiderocol [12].

In the study conducted by Falagas, cefiderocol achieved an MIC90 of $0.5 \mathrm{mg} / \mathrm{L}$ against meropenem intermediate 
isolates and MIC90 of $1 \mathrm{mg} / \mathrm{L}$ against meropenem-resistant isolates of Enterobacteriaceae, A. baumannii, P. aeruginosa, $P$. stuartii, K. pneumoniae, and E. cloacae, which was superior to tigecycline and colistin [13]. Again, the potent antibacterial activity of cefiderocol was confirmed with MICs less than $4 \mu \mathrm{g} / \mathrm{mL}$ against isolates of GNB that are not susceptible to ceftazidime-avibactam, ceftolozane-tazobactam, and colistin [7, 11]. Against meropenem susceptible isolates from North America and Europe, cefiderocol exhibited an MIC90 of 0.5 and $1 \mathrm{mg} / \mathrm{L}$ for Enterobacteriaceae; 0.5 and $0.5 \mathrm{mg} / \mathrm{L}$ for $P$. aeruginosa; 1 and $2 \mathrm{mg} / \mathrm{L}$ for Acinetobacter spp.; 0.5 and $0.25 \mathrm{mg} / \mathrm{L}$ for $S$. maltophilia; 0.12 and 0.5 $\mathrm{mg} / \mathrm{L}$ for B. cepacia complex spp., respectively [7]. Against meropenem-resistant isolates, cefiderocol had an MIC $90 \leq 4$ $\mathrm{mg} / \mathrm{L}$ for $99.6 \%$ (245/246) of Enterobacteriaceae, $99.7 \%$ (394/395) of P. aeruginosa, 96.1\% (540/562) of Acinetobacter spp., and $87.1 \%$ (27/31) of B. cepacia complex spp. [7].

As part of SIDERO-WT-2014 surveillance study, isolates of carbapenemase-producing, and carbapenemase-negative and meropenem-resistant isolates of Enterobacteriaceae, $P$. aeruginosa, A. baumannii were tested for susceptibility to cefiderocol [14]. Cefiderocol suppressed the growth of $97.7 \%$ of isolates with an MIC less than $4 \mu \mathrm{g} / \mathrm{mL}$, including those carrying additional ESBL or AmpC producing GNB [14]. The study results confirmed that cefiderocol MIC elevation is not necessarily due to carrying beta-lactamases but possibly related to the disruption of iron transport proteins or upregulation of efflux transporter [14]. Cefiderocol has demonstrated excellent activity in Gram-negative organisms including carbapenemase-producing organisms. Also, in vitro studies showed the superiority of cefiderocol to currently available antibiotics, including new agents such as ceftazidime-avibactam, ceftolozane-tazobactam, and colistin. However, cefiderocol is not active against Gram-positive bacteria and anaerobes [14]. Detailed study information is available in Table 3.

\subsection{Animal Studies}

A rat respiratory tract infection model was used to evaluate the antibacterial efficacy of cefiderocol that simulates human pharmacokinetic profiles [25]. Unlike murine thigh infection models, this model utilizes the immunocompetent rat and requires longer treatment of 4 days to eradicate bacterial infections [25]. Administering cefiderocol $2 \mathrm{~g}$ every $8 \mathrm{~h}$ as a 3-h infusion for 4 days achieved a greater than 3 $\log 10$ reduction in cells of carbapenem-resistant isolates of $P$. aeruginosa, A. baumannii, and $K$. pneumoniae in lung infection [25]. As with other beta-lactam antibiotics, the efficacy of cefiderocol correlates closely with the time that the free drug concentration is above the MIC ( $\% \mathrm{fT}>\mathrm{MIC}$ ) $[25,26]$. Prolonged 3-h infusion of cefiderocol maintained $100 \% \mathrm{fT}>$ MIC for MIC of $4 \mu \mathrm{g} / \mathrm{mL}$ as compared to 75

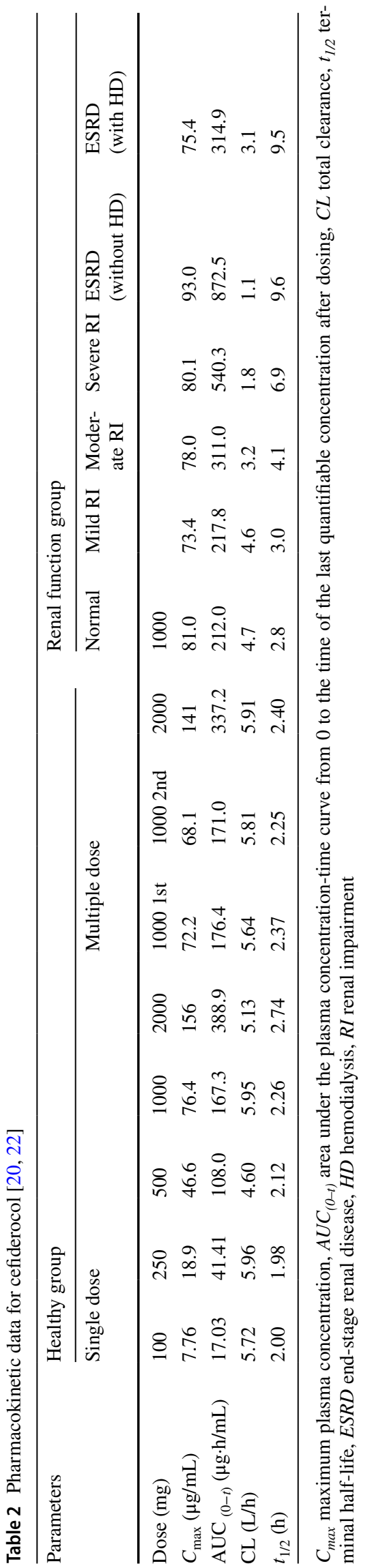


\%fT $>$ MIC with a 1-h infusion [25]. This study suggests the use of cefiderocol in the treatment of lung infections caused by carbapenem-resistant GNB [25]. Another study described the pharmacokinetics, pharmacodynamics, and 24-h efficacy of cefiderocol in a neutropenic murine thigh infection model [27]. Six neutropenic mice were infected with $P$. aeruginosa to estimate pharmacokinetic parameters using mean serum concentration data, which showed a mean $\mathrm{t}_{1 / 2}$ of $0.86 \mathrm{~h}$ and similar pharmacokinetics in the range of doses tested (4, 100 , and $250 \mathrm{mg} / \mathrm{kg}$ ) with a one-compartment model [27]. In pharmacodynamic assessments, the efficacy of cefiderocol was investigated based on \% fT $>$ MIC and the change of bacterial density expressed in $\log 10 \mathrm{CFU} / \mathrm{mL}$ after 24-h exposures [27]. The pharmacodynamics of cefiderocol showed sigmoidal dose-response curves as greater bacterial killing is achieved with increasing doses [27]. The study demonstrated that antibacterial effects were observed with $\% \mathrm{fT}>$ MIC greater than $80 \%$, as evidenced by $76.3,81.9$, and $88.2 \%$ to result in bacterial stasis, $1 \log 10$, and $2 \log 10$ CFU reductions, respectively [27]. Unlike other siderophore beta-lactams, cefiderocol produced a sustained bacterial killing with $2 \log 10 \mathrm{CFU}$ reductions in 24-h treatment against $P$. aeruginosa [27]. Furthermore, cefiderocol exhibited potent activity against all $P$. aeruginosa isolates, which were resistant to cefepime and levofloxacin [27].

The third study investigated the in vivo efficacy of cefiderocol against Enterobacteriaceae and $P$. aeruginosa in neutropenic thigh models and Enterobacteriaceae, P. aeruginosa, A. baumannii, and S. maltophilia in lung infection models [26]. Compared to cefepime, which showed $\% \mathrm{fT}>\mathrm{MIC}$ of $61.7 \%$ and $87.7 \%$ to reach a bacterial stasis and a $1 \log 10 \mathrm{CFU}$ reduction, cefiderocol demonstrated lower values of \%fT > MIC of $47.5 \%$ and $57.6 \%$, respectively [26]. To achieve a $1-\log 10$ reduction, $\% \mathrm{fT}>$ MIC for Enterobacteriaceae and $P$. aeruginosa in the thigh infection models were $73.3 \%$ and $77.2 \%$, respectively [26]. Against Enterobacteriaceae, $P$. aeruginosa, A. baumannii, and $S$. maltophilia in the lung infection model, \%fT $>$ MIC were $64.4 \%, 70.3 \%, 88.1 \%$, and $53.9 \%$, respectively [26]. A. baumannii required higher \%fT $>$ MIC values than those for Enterobacteriaceae and $P$. aeruginosa, which demonstrated similar efficacy in both thigh and lung infection models [26]. These results indicate cefiderocol as being the treatment option for MDR Gram-negative bacterial infections [25-27].

\subsection{Clinical Trials}

The phase II, randomized, double-blind, parallel-group non-inferiority trial (APEKS-cUTI) evaluated the efficacy and safety of cefiderocol against imipenem-cilastatin for the treatment of cUTIs caused by GNB [28]. This study included hospitalized patients $\geq 18$ years old who met the FDA diagnostic criteria for UTI and diagnosed with cUTI with or without pyelonephritis, or acute uncomplicated pyelonephritis, including immunosuppressed patients with renal transplant [28]. Patients with acute uncomplicated pyelonephritis were limited to $30 \%$ in this study to assess more complicated MDR infections in older patients with complex co-morbidities, including renal impairment [28]. 448 patients were randomized $2: 1$ to receive either cefiderocol $2 \mathrm{~g}$ or imipenem-cilastatin $1 \mathrm{~g} / 1 \mathrm{~g} 1$-h intravenous infusion every $8 \mathrm{~h}$ for 7-14 days with renally adjusted doses [28].

Uropathogens, including E. coli, K. pneumoniae, P. aeruginosa, Proteus mirabilis, Enterobacter cloacae, Morganella morganii, and Citrobacter freundii, have different resistance patterns against antibiotics classes [28]. The most common pathogens were beta-lactamase producing $E$. coli, which are extensively drug-resistant and pandrug-resistant bacteria, and the leading cause of community-acquired and hospital-acquired infections [28].

At 7 days after the end of treatment, $183(73 \%)$ of 252 patients in the cefiderocol group and 65 (55\%) of 119 patients in the imipenem-cilastatin group achieved the composite outcome of clinical and microbiological response in the modified intention-to-treat population (mITT) [treatment difference of $18.58 \%$; 95\% CI 8.23-28.92, $p=0.0004$, number needed to treat $(\mathrm{NNT})=6$ ] [28]. This difference is clinically meaningful since it is consistent within all efficacy populations and resulted from microbiological eradication of GNB to $<10^{4} \mathrm{CFU} / \mathrm{mL}$ from initial $\geq 10^{5} \mathrm{CFU} / \mathrm{mL}$ [28]. Therefore, cefiderocol was non-inferior to imipenem-cilastatin for primary endpoints [28]. Notably, cefiderocol demonstrated superiority to imipenem-cilastatin for the composite primary outcome in the post hoc analysis [28].

The pathogen-specific, randomized, prospective, phase III clinical study, the CREDIBLE-CR study, was conducted to investigate the efficacy and safety of cefiderocol for the treatment of carbapenem-resistant (CR) Gram-negative infections [29]. According to European Medicines Agency (EMA), patients with a confirmed diagnosis of HAP/VAP/ HCAP, BSI or sepsis, or cUTI caused by CR Gram-negative infections, were randomized 2:1 to receive $2 \mathrm{~g}$ of cefiderocol every $8 \mathrm{~h}$ for a 3-h infusion or best available therapy (BAT) [29]. BAT as defined by a maximum of three antibiotics in combination or subsequent de-escalation at the early assessment (EA) time point was selected by the site investigator, considering the clinical condition of the patient [29].

The treatment duration for HAP/VAP/HCAP or BSI/ sepsis was 7-14 days and 5 days for cUTIs, and can be extended to 21 days to achieve clinical cure [29]. The primary endpoints included clinical cure rates at the time of cure (TOC) in patients with HAP/VAP/HCAP, BSI or sepsis, and microbiological outcomes at TOC in patients with cUTI in the CR-micro intention-to-treat (ITT) population [29]. Secondary endpoints of the study were clinical outcome at the end of therapy (EOT) and follow-up (FUP) time 


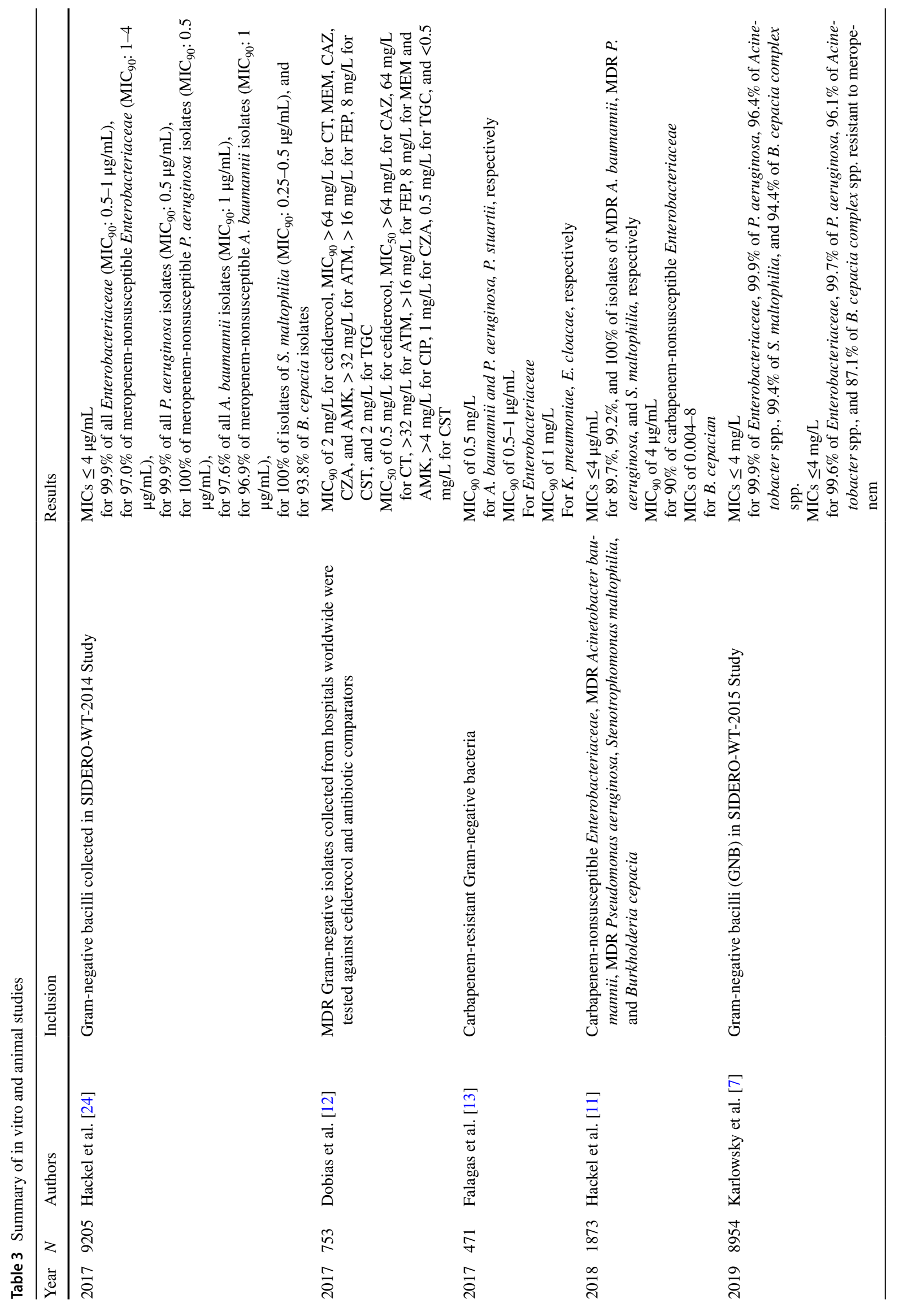




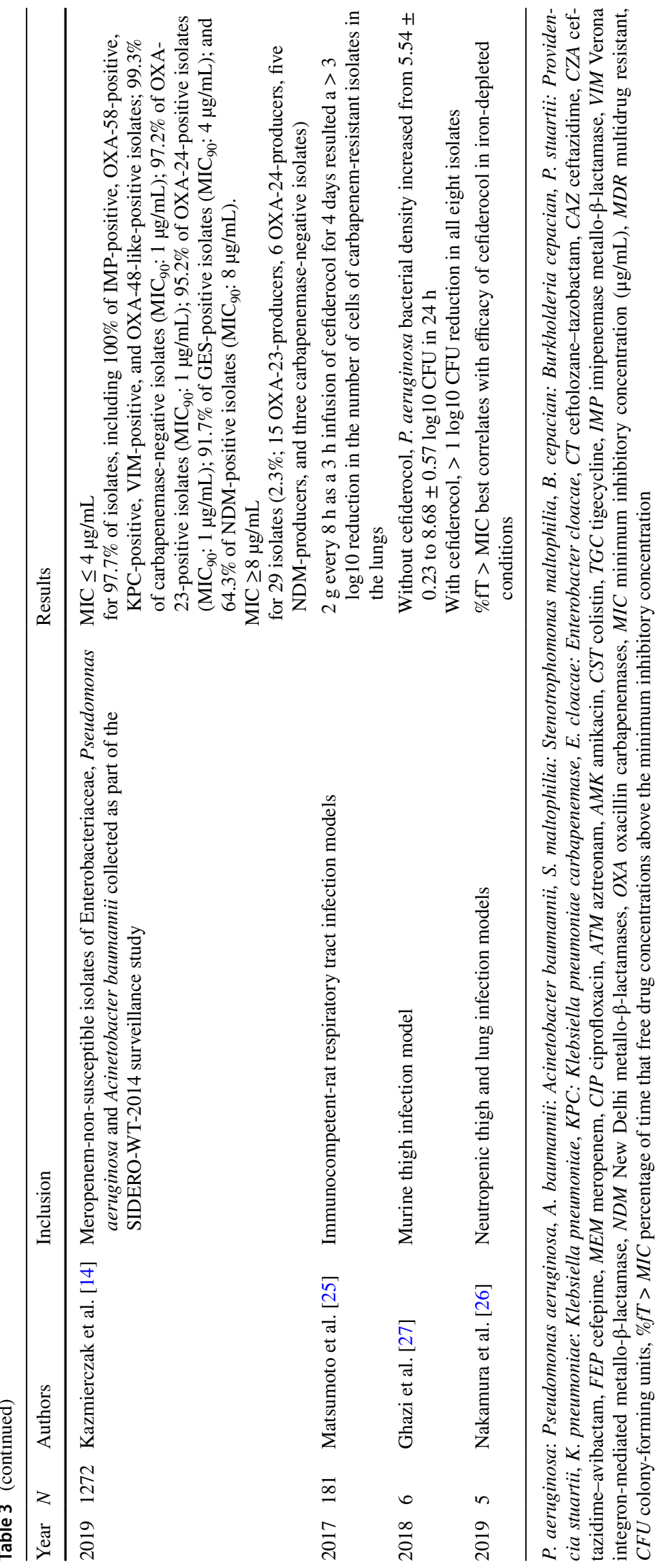


points and microbiological outcomes (EOT, TOC, FUP), allcause mortality in patients with HAP/VAP/HCAP, BSI or sepsis at days 14 and 28, as well as microbiological and clinical outcome (EOT, TOC, FUP), and composite clinical and microbiological outcome (EOT, TOC, FUP) for patients with cUTIs [29]. Safety and pharmacokinetic parameters of cefiderocol and BAT were assessed [29]. The all-cause mortality was higher in the cefiderocol group $(18.0 \%, 24.8 \%$, and $33.7 \%)$ versus the BAT group $(12.2 \%, 18.4 \%$, and $18.4 \%)$ at Days 14, 28, and end of study (EOS), respectively [30].

Another phase III clinical trial (APEKS-NP study) investigated cefiderocol versus meropenem against GNB for the treatment of hospital-acquired bacterial pneumonia, ventilator-associated bacterial pneumonia, or healthcare-associated bacterial pneumonia [31]. The primary endpoint was allcause mortality at 14 days after administering $2 \mathrm{~g}$ of cefiderocol or meropenem every $8 \mathrm{~h}$ for 7-14 days intravenously and $600 \mathrm{mg}$ of linezolid intravenously every $12 \mathrm{~h}$ at least for 5 days [31]. The all-cause mortality was $12.4 \%, 21.2 \%$, and $26.9 \%$ for cefiderocol versus $11.6 \%, 20.1 \%$, and $22.8 \%$ for meropenem at Day 14, Day 28, and EOS, respectively [30].

Hence, there are safety concerns in the treatment of pneumonia with increased all-cause mortality related to cefiderocol, as evidenced by both the CREDIBLE-CR study and the APEKS-NP study [29-31].

A phase II clinical trial for bloodstream infection versus the best available therapy is recruiting now.

\subsection{Safety}

The phase I study evaluated the safety, tolerability, and pharmacokinetics of cefiderocol in patients with renal impairment after administering a single 1000-mg intravenous infusion over 10 days [22]. There was no mortality or serious adverse events (AEs) in 38 subjects [22]. One patient with moderate renal impairment had to withdraw from the medication due to urticaria with underlying lactose intolerance and seasonal allergies [22]. This urticaria was unrelated to cefiderocol since no antibodies against cefiderocol were detected in any blood samples [22]. The most common AE was mild contact dermatitis (7.9\%) in one patient in each mild, severe, and end-stage renal disease (ESRD) group followed by mild nausea in one patient with moderate disease and ESRD without hemodialysis [22].

The phase II, randomized, double-blind, parallel-group non-inferiority trial (APEKS-cUTI) also evaluated safety, clinical responses assessed by the investigator, and microbiological responses evaluated by quantitative urinalysis early, at TOC, and at14 days after the end of treatment [28]. The safety of cefiderocol was conducted for an extended treatment period without having confounding factors such as step-down oral treatment [28]. AEs reported were 122 $(41 \%)$ of 300 patients in the cefiderocol group and $76(51 \%)$ of 148 patients in the imipenem-cilastatin group [28]. The most common AEs in both treatment groups involved gastrointestinal symptoms such as diarrhea, constipation, nausea, vomiting, or upper abdominal pain (35 [12\%] patients in the cefiderocol group and 27 [18\%] patients in the imipenem-cilastatin group) [28]. However, there was less incidence of diarrhea and $C$. difficile infection in the cefiderocol group $(4 \% ; 13 / 300)$ compared to the imipenem-cilastatin group $(6 \% ; 9 / 148)$ since cefiderocol does not have activity against anaerobes or Gram-positive bacteria, preventing the increased risk of $C$. difficile colitis by using broad-spectrum imipenem [28].

Still, there is a possibility of developing $C$. difficileassociated diarrhea (CDAD) with any systemic antibiotics, including cefiderocol, ranging from mild diarrhea to fatal colitis that may require colectomy [8]. Beta-lactam antibacterial drugs can cause hypersensitivity reactions, including serious skin reactions to life-threatening anaphylactic reactions [8]. Therefore, care must be taken before starting cefiderocol for patients with the previous history of allergic reactions to beta-lactams such as penicillin, cephalosporins, or other beta-lactam antibiotics [8]. Special precautions are required for the use of cephalosporins, such as cefiderocol in patients with a history of epilepsy or renal impairment to prevent seizures and other central nervous system adverse reactions [8]. Since there is limited information about cerebrospinal fluid (CSF) penetration of cefiderocol available, we were not able to find the CSF penetration information.

In the CREDIBLE-CR study, all-cause mortality was increased in critically ill patients who have carbapenemresistant GNB infections such as nosocomial pneumonia, bloodstream infections, or sepsis, and treated with cefiderocol compared to BAT $[8,29]$. The all-cause mortality in the cefiderocol group was higher than the BAT group through Day 28 and Day 49, as evidenced by [25/101 (24.8\%) vs. 9/49 (18.4\%), treatment difference $6.4 \%, 95 \%$ CI $(-8.6$, $19.2)]$, and [34/101 (33.7\%) vs. $10 / 49(20.4 \%)$, treatment difference $13.3 \%, 95 \% \mathrm{CI}(-2.5,26.9)]$, respectively [8, 29]. The main cause of death was the worsening of underlying diseases or infections caused by nonfermenters such as $A$. baumannii, S. maltophilia, and P. aeruginosa; however, the cause of the higher mortality is still unclear $[8,29]$.

\section{Place in Therapy}

The WHO defines priority 1 pathogens as critical for research and development of new antibiotic drugs to treat carbapenem-resistant A. baumannii, P. aeruginosa, and carbapenem-resistant or third-generation cephalosporin-resistant Enterobacteriaceae (CRE) [32]. CDC emphasizes CRE infections in healthcare settings due to its high mortality rates associated with bloodstream infections and high levels 
of resistance to many antibiotic options [2]. CRE infections include bloodstream infections, ventilator-associated pneumonia, intra-abdominal abscesses, and most commonly urinary tract infections from urinary retention or a urinary catheter [1].

Cefiderocol (Fetroja ${ }^{\circledR}$ ) received FDA approval on 14 November 2019 for the treatment of cUTIs such as pyelonephritis for patients 18 years and older due to Gram-negative pathogens, including Escherichia coli, Klebsiella pneumoniae, Proteus mirabilis, Pseudomonas aeruginosa, and Enterobacter cloacae complex [8]. To have potent efficacy of cefiderocol without developing the risk of antimicrobial resistance, its use should be limited to treat or prevent proven and probable infections caused by GNB listed above [8].

Since the safety and efficacy of cefiderocol for the treatment of nosocomial pneumonia, bloodstream infections, or sepsis need further investigation, cefiderocol use should be reserved for cUTI treatment if no other alternatives are available $[8,29,31]$.

The recommended dosing for cUTIs is $2 \mathrm{~g}$ IV over 3 $\mathrm{h}$ at 8 -h intervals for 7-14 days with creatinine clearance (CLcr) of $60-119 \mathrm{~mL} / \mathrm{min}$ [8]. Dose adjustment is required in patients with renal impairment if CLcr is less than 60 $\mathrm{mL} / \mathrm{min}$ or greater than $120 \mathrm{~mL} / \mathrm{min}$ [8]. Patients with CLcr $30-59 \mathrm{~mL} / \mathrm{min}$ should receive $1.5 \mathrm{~g} \mathrm{IV}$ over $3 \mathrm{~h}$ at 8 -h intervals, and patients with CLcr $15-29 \mathrm{~mL} / \mathrm{min}$ should receive $1 \mathrm{~g} \mathrm{IV}$ over $3 \mathrm{~h}$ at 8 -h intervals, and ESRD patients with CLcr $15 \mathrm{~mL} / \mathrm{min}$ or less should receive $0.75 \mathrm{~g}$ over $3 \mathrm{~h}$ at 12-h intervals [8]. If CLcr is $120 \mathrm{~mL} / \mathrm{min}$ or greater, patients should receive $2 \mathrm{~g}$ IV over $3 \mathrm{~h}$ at 6 -h intervals [8]. The current average wholesale price (AWP) for the current standard of therapy of imipenem/cilastatin is approximately US $\$ 135$ per IV patient treatment day, and the AWP for imipenem/ cilastatin/relebactam, ceftazidime/avibactam, and cefiderocol is approximately US\$1284, \$1292, and \$1320, respectively, per IV patient treatment day [33-36].

\section{Conclusion}

Cefiderocol has demonstrated excellent activity against GNB, including Enterobacteriaceae, P. aeruginosa, A. baumannii, B. cepacia, and S. maltophilia.

Until further investigation is completed in other MDR Gram-negative infections, cefiderocol should be considered only as a treatment option for the treatment of cUTIs from GNB, especially in patients who have limited or no alternative treatment options.

\section{Compliance with Ethical Standards}

Funding No funding was received to prepare this review.
Conflict of interest The authors declare that they have no conflict of interest.

Research involving human participants and/or animals For this type of review formal consent is not required.

\section{References}

1. CDC. Carbapenem-resistant Enterobacteriaceae (CRE) Infection: Clinician FAQs. CDC. 2019. https://www.cdc.gov/hai/organisms/ cre/cre-clinicians.html. Accessed 6 Mar 2020.

2. CDC. Guidance for Control of Carbapenem-resistant Enterobacteriaceae (CRE). CDC 2012 PDF. 2012. https://www.cdc.gov/hai/ pdfs/cre/cre-guidance-508.pdf. Accessed 6 Mar 2020.

3. US Department of Health and Human Services, Food and Drug Administration, Center for Drug Evaluation and Research. Antibacterial therapies for patients with unmet medical need for the treatment of serious bacterial diseases. Guidance for industry. 2017. https://www.fda.gov/downloads/Drugs/Guidances/UCM35 9184.pdf. Accessed 6 Mar 2020.

4. Ito A, Sato T, Ota M, et al. In vitro antibacterial properties of cefiderocol, a novel siderophore cephalosporin, against Gramnegative bacteria. Antimicrob Agents Chemother. 2017;62:1. https ://doi.org/10.1128/AAC.01454-17.

5. Aoki T, Yoshizawa H, Yamawaki K, et al. Cefiderocol (S-649266), a new siderophore cephalosporin exhibiting potent activities against Pseudomonas aeruginosa and other gram-negative pathogens including multi-drug resistant bacteria: structure activity relationship. Eur J Med Chem. 2018;155:847-68. https://doi. org/10.1016/j.ejmech.2018.06.014 (Epub 2018 Jun 8).

6. Ito A, Kohira N, Bouchillon SK, et al. In vitro antimicrobial activity of S-649266, a catechol-substituted siderophore cephalosporin, when tested against non-fermenting Gram-negative bacteria. J Antimicrob Chemother. 2016;71(3):670-7. https://doi. org/10.1093/jac/dkv402 (Epub 2015 Dec 7).

7. Karlowsky JA, Hackel MA, Tsuji M, et al. In vitro activity of cefiderocol, a siderophore cephalosporin, against Gram-negative bacilli isolated by clinical laboratories in North America and Europe in 2015-2016: SIDERO-WT-2015. Int J Antimicrob Agents. 2019;53(4):456-66. https://doi.org/10.1016/j.ijantimica g.2018.11.007 (Epub 2018 Nov 22).

8. Fetroja (Cefiderocol) [prescribing information]. Osaka JSC, Ltd. 2020. https://www.accessdata.fda.gov/drugsatfda_docs/label /2019/209445s000lbl.pdf. Accessed 6 Mar 2020.

9. Ito A, Nishikawa T, Matsumoto S, et al. Siderophore cephalosporin cefiderocol utilizes ferric iron transporter systems for antibacterial activity against Pseudomonas aeruginosa. Antimicrob Agents Chemother. 2016;60(12):7396-401. https://doi. org/10.1128/AAC.01405-16.

10. Ito-Horiyama T, Ishii Y, Ito A, et al. Stability of novel siderophore cephalosporin S-649266 against clinically relevant carbapenemases. Antimicrob Agents Chemother. 2016;60(7):4384-6. https://doi.org/10.1128/AAC.03098-15.

11. Hackel MA, Tsuji M, Yamano Y, et al. In vitro activity of the siderophore cephalosporin, cefiderocol, against carbapenemnonsusceptible and multidrug-resistant isolates of Gram-negative bacilli collected worldwide in 2014 to 2016. Antimicrob Agents Chemother. 2018;62:2. https://doi.org/10.1128/AAC.01968-17.

12. Dobias J, Dénervaud-Tendon V, Poirel L, Nordmann P. Activity of the novel siderophore cephalosporin cefiderocol against multidrug-resistant Gram-negative pathogens. Eur J Clin Microbiol Infect Dis. 2017;36(12):2319-27. https://doi.org/10.1007/s1009 6-017-3063-z (Epub 2017 Jul 26). 
13. Falagas ME, Skalidis T, Vardakas KZ, Legakis NJ. Activity of cefiderocol (S-649266) against carbapenem-resistant Gram-negative bacteria collected from inpatients in Greek hospitals. J Antimicrob Chemother. 2017;72(6):1704-8. https://doi.org/10.1093/ $\mathrm{jac} / \mathrm{dkx} 049$.

14. Kazmierczak KM, Tsuji M, Wise MG, et al. In vitro activity of cefiderocol, a siderophore cephalosporin, against a recent collection of clinically relevant carbapenem-non-susceptible Gramnegative bacilli, including serine carbapenemase- and metallo$\beta$-lactamase-producing isolates (SIDERO-WT-2014 Study). Int J Antimicrob Agents. 2019;53(2):177-84. https://doi.org/10.1016/j. ijantimicag.2018.10.007 (Epub 2018 Oct 26).

15. Jacobs MR, Abdelhamed AM, Good CE, et al. ARGONAUT-I: activity of cefiderocol (S-649266), a siderophore cephalosporin, against Gram-negative bacteria, including carbapenem-resistant nonfermenters and Enterobacteriaceae with defined extendedspectrum-lactamases and carbapenemases. Antimicrob Agents Chemother. 2018;63:1. https://doi.org/10.1128/AAC.01801-18.

16. Delgado-Valverde M, Conejo MDC, Serrano L, FernándezCuenca F, Pascual Á. Activity of cefiderocol against high-risk clones of multidrug-resistant Enterobacterales, Acinetobacter baumannii, Pseudomonas aeruginosa and Stenotrophomonas maltophilia. J Antimicrob Chemother. 2020;2020:dka117. https ://doi.org/10.1093/jac/dkaa117.

17. Golden AR, Adam HJ, Baxter M, et al. In vitro activity of cefiderocol, a novel siderophore cephalosporin, against Gram-negative Bacilli isolated from patients in Canadian intensive care units. Diagn Microbiol Infect Dis. 2020;97(1):115012. https://doi. org/10.1016/j.diagmicrobio.2020.115012.

18. Rolston KVI, Gerges B, Shelburne S, et al. Activity of cefidero$\mathrm{col}$ and comparators against isolates from cancer patients. Antimicrob Agents Chemother. 2020;64(5):e01955-19. https://doi. org/10.1128/AAC.01955-19.

19. Kawai A, et al. Structural basis of reduced susceptibility to ceftazidime-avibactam and cefiderocol in Enterobacter cloacae due to AmpC R2 loop deletion. Antimicrob Agents Chemother. 2020. https://doi.org/10.1128/AAC.00198-2.

20. Saisho Y, Katsube T, White S, Fukase H, Shimada J. Pharmacokinetics, safety, and tolerability of cefiderocol, a novel siderophore cephalosporin for Gram-negative bacteria, in healthy subjects. Antimicrob Agents Chemother. 2018;62:3. https://doi. org/10.1128/AAC.02163-17.

21. Miyaaki S, Katsube T, Shen H, Tomek C, Narukawa Y. Metabolism, excretion, and pharmacokinetics of [14C]-cefiderocol (S-649266), a siderophore cephalosporin, in healthy subjects following intravenous administration. J Clin Pharmacol. 2019;59(7):958-67. https://doi.org/10.1002/jcph.1386 (Epub 2019 Feb 7).

22. Katsube T, Echols R, Arjona Ferreira JC, et al. Cefiderocol, a siderophore cephalosporin for Gram-negative bacterial infections: pharmacokinetics and safety in subjects with renal impairment. J Clin Pharmacol. 2017;57(5):584-91. https://doi.org/10.1002/ jcph.841 (Epub 2016 Nov 22).

23. Katsube T, Wajima T, Ishibashi T, Arjona Ferreira JC, Echols R. Pharmacokinetic/pharmacodynamic modeling and simulation of cefiderocol, a parenteral siderophore cephalosporin, for dose adjustment based on renal function. Antimicrob Agents Chemother. 2016;61:1. https://doi.org/10.1128/AAC.01381-16.

24. Hackel MA, Tsuji M, Yamano Y, et al. In vitro activity of the siderophore cephalosporin, cefiderocol, against a recent collection of clinically relevant Gram-negative bacilli from North America and Europe, including carbapenem-nonsusceptible isolates (SIDERO-WT-2014 Study). Antimicrob Agents Chemother. 2017;61:9. https://doi.org/10.1128/AAC.00093-17.

25. Matsumoto S, Singley CM, Hoover J, et al. Efficacy of cefiderocol against carbapenem-resistant Gram-negative bacilli in immunocompetent-rat respiratory tract infection models recreating human plasma pharmacokinetics. Antimicrob Agents Chemother. 2017;61:9. https://doi.org/10.1128/AAC.00700-17.

26. Nakamura $R$, Ito-Horiyama $T$, Takemura $M$, et al. In vivo pharmacodynamic study of cefiderocol, a novel parenteral siderophore cephalosporin, in murine thigh and lung infection models. Antimicrob Agents Chemother. 2019;63:9. https://doi.org/10.1128/ AAC.02031-18.

27. Ghazi IM, Monogue ML, Tsuji M, Nicolau DP, et al. Pharmacodynamics of cefiderocol, a novel siderophore cephalosporin, in a Pseudomonas aeruginosa neutropenic murine thigh model. Int $\mathbf{J}$ Antimicrob Agents. 2018;51(2):206-12. https://doi.org/10.1016/j. ijantimicag.2017.10.008 (Epub 2017 Oct 27).

28. Portsmouth S, Veenhuyzen DV, Echols R, et al. Cefiderocol versus imipenem-cilastatin for the treatment of complicated urinary tract infections caused by Gram-negative uropathogens: a phase 2, randomised, double-blind, non-inferiority trial. Lancet Infect Dis. 2018;18(12):1319-28. https://doi.org/10.1016/S1473 -3099(18)30554-1.

29. Bassetti M, Ariyasu M, Binkowitz B, et al. Designing a pathogenfocused study to address the high unmet medical need represented by carbapenem-resistant Gram-negative pathogens-the international, multicenter, randomized, open-label, phase 3 CREDIBLE-CR study. Infect Drug Resist. 2019;12:3607-23. https://doi. org/10.2147/IDR.S225553.

30. FDA Antimicrobial Drugs Advisory Committee, Cefiderocol Briefing Document NDA \#209445. Shionogi Inc. https://www. fda.gov/media/131705/download. Accessed 27 May 2020.

31. Clinical Study of S-649266 for the Treatment of Nosocomial Pneumonia Caused by Gram-negative Pathogens (APEKS-NP). https://clinicaltrials.gov/ct2/show/NCT03032380?term=NCT03 032380\&draw $=2 \&$ rank $=1$. Accessed 23 Feb 2020.

32. World Health Organization (WHO). Global priority of antibioticresistant bacteria to guide research, discovery, and development of new antibiotics. 2017. https://www.who.int/medicines/publi cations/WHO-PPL-Short_Summary_25Feb-ET_NM_WHO.pdf. Accessed 24 Feb 2020.

33. Imipenem and Cilastatin. In: Lexi-Drugs [database online]. Hudson, Ohio: Wolters-Kluwer Health. 2019. Accessed 23 Feb 2020.

34. Imipenem, cilastatin, and relebactam. In: Lexi-Drugs [database online]. Hudson, Ohio: Wolters-Kluwer Health. 2019. Accessed 23 Feb 2020.

35. Ceftazidime and Avibactam In: Lexi-Drugs [database online]. Hudson, Ohio: Wolters-Kluwer Health. 2019. Accessed 23 Feb 2020.

36. Cefiderocol In: Lexi-Drugs [database online]. Hudson, Ohio: Wolters-Kluwer Health. 2019. Accessed 23 Feb 2020. 